\author{
BY W. J. BüHLER
}

STATISTICAL LABORATORY, UNIVERSITY OF CALIFORNIA, BERKELEY, AND DEPARTMENT OF MATHEMATICS, UNIVERSITY OF HEIDELBERG, GERMANY

Communicated by Jerzy Neyman, October 21, 1966

1. Introduction.-The usual definition of probability generating functions applies to nonnegative integer-valued random variables. In this paper the term probability generating function (pgf) of a random variable $X$ will be used to denote the function

$$
f(s)=E s^{x}=\int_{0}^{\infty} s^{x} d F(x)
$$

where $X$ is nonnegative, but not necessarily integer-valued, and where $F$ denotes the distribution function of $X$. The paper deals with properties of (1.1) in their relation to probabilistic properties of $X$.

2. Types of Probability Generating Functions.-For $0 \leqq s \leqq 1$, the probability generating function $f$ is a real-valued nondecreasing function of its argument with $0 \leqq f(0) \leqq f(1)=1$. It is well known that, for $X$ integer-valued, the function $f$ is convex. In general, this remains true only as long as $P(0<X<1)=0$; more precisely, for any positive integer $k$ we have

Lemma 2.1. If $P(0<X<k-1, X$ not integer $)=0$, then

(i) $f^{(j)}(0)=j ! P(X=j) \geqq 0 ; j=0,1,2, \ldots, k-1$

(ii) $f^{(j)}(s)$ is nonnegative and nondecreasing for

$$
j=0,1, \ldots, k-1 ; 0<s .
$$

Furthermore, if $P(k-1<X<k)>0$, then

(iii) $f^{(k)}(0)=+\infty, f^{(k)}(s) \geqq 0,0<s$.

Proof: To show (i) and the first part of (iii) we write (1.1) as

$$
f(s)=\sum_{i=0}^{k-1} p_{i} s^{i}+\int_{(k-1)^{+}}^{\infty} s^{x} d F(x), \text { where } p_{i}=P(X=i) .
$$

Thus, for $j=0,1, \ldots, k-1$,

$$
\begin{aligned}
f^{(j)}(0) & =j ! p_{j}+\lim _{s \rightarrow 0} \frac{1}{s^{j}} \sum_{i=0}^{j}(-1)^{(j-i)}\left(\begin{array}{l}
j \\
i
\end{array}\right) \int_{(k-1)^{+}}^{\infty}(i s)^{x} d F(x) \\
& =j ! p_{j}+\lim _{s \rightarrow 0} \sum_{i=0}^{j}(-1)^{(j-i)}\left(\begin{array}{l}
j \\
i
\end{array}\right) \int_{(k-1)^{+}}^{\infty} i^{x} s^{(x-j)} d F(x) .
\end{aligned}
$$

Each of the integrands being bounded and monotone decreasing to zero assertion $(i)$ follows. For $j=k$, however, we obtain

$$
f^{(k)}(0)=\lim _{s \rightarrow 0} \sum_{i=0}^{k}(-1)^{(k-i)}\left(\begin{array}{c}
k \\
i
\end{array}\right) \int_{(k-1)^{+}}^{\infty} i^{x} s^{(x-k)} d F(x) .
$$

Since $P(k-1<X<k-1+a)>0$ for some $a<1$, one has, as $s \rightarrow 0$, 


$$
\begin{aligned}
\int_{(k-1)^{+}}^{\infty} i^{x} s^{(x-k)} d F(x) \geq \int_{(k-1)^{+}}^{k-1+a} i^{x} s^{(x-k)} d F(x) \\
\quad \geq i^{(k-1)} s^{a-1} P(k-1<X<k-1+a) \rightarrow \infty
\end{aligned}
$$

which proves the first part of (iii). Assertion (ii) and the second part of (iii) can be shown by differentiating (1.1) under the integral sign. It is seen that $f^{(j)}(s)$ is even strictly positive unless with probability one $X$ takes only the values $0,1, \ldots$, $j-1$. In particular, $P(0<X<1)=0$ and $P(X>1)>0$ imply that $f(s)$ is strictly convex. Thus, in this case the equation $f(s)=s$ can have at most one solution $q$ in the open unit interval. In the general case, this will be true as a consequence of the following lemma.

Lemma 2.2. If $P(X=0$ or $X=1)<1$, then the equation $f^{\prime \prime}(s)=0$ has at most one solution $\rho>0$.

Proof: Let $0<s<t$. Then, for $0<x \neq 1$ we have $x(x-1) s^{x}<x(x-1) t^{x} s / t$, and therefore the relation

$$
s^{2} f^{\prime \prime}(s)=\int_{0}^{\infty} x(x-1) s^{x} d F(x)<\frac{s}{t} \int_{0}^{\infty} x(x-1) t^{x} d F(x)=s t f^{\prime \prime}(t),
$$

which shows that not both $f^{\prime \prime}(s)$ and $f^{\prime \prime}(t)$ can be zero.

Differentiating (1.1) under the integral sign at $s=1$ (taking left derivatives if needed), we obtain

Lemma 2.3. The kkth factorial moment $\operatorname{EX}(X-1) \ldots(X-k+1)$ of $X$ is given by

$$
\mu_{k}=f^{(k)}(1)
$$

which may be finite or not.

Let $p=P(X=0)$ and $m=\mu_{1}=E X$; then we can summarize the results ob-

\begin{tabular}{|c|c|c|c|}
\hline Linear & $\begin{array}{l}0-\text { Type }(p=0) \\
m=1\end{array}$ & $\begin{array}{c}p \text {-Type }(0<p<1) \\
m=1-p<1\end{array}$ & $\begin{array}{c}\text { Trivial }(p=1) \\
m=0\end{array}$ \\
\hline Convex & $m>1$ & $\left\{\begin{array}{r}1-p<m<1 \\
m=1 \\
m>1\end{array}\right.$ & \\
\hline Concave & $m<1$ & $m<1$ & \\
\hline$\rho-T y p e$ & $\begin{array}{l}\left\{\begin{array}{l}m<1 \\
m=1 \\
m>1\end{array}\left\{\begin{array}{l}\rho<q \\
\rho=q \\
\rho>q\end{array}\right.\right.\end{array}$ & $\begin{array}{l}\left\{\begin{array}{l}m<1 \\
m=1 \\
m>1\end{array}\left\{\begin{array}{l}\rho<q \\
\rho=q \\
\rho>q\end{array}\right.\right.\end{array}$ & \\
\hline
\end{tabular}
tained so far:

TheOREM 2.1. The probability generating function $f(s), 0 \leq s \leq 1$, of a distribution on $[0, \infty[$ is of one of the types listed below and drawn in Figure 1:

In fact, any function not of one of the types listed has been excluded by Lemma 2.1 or Lemma 2.2.

ThEOREM 2.2. There are probability generating functions of any of the types listed in Theorem 2.1. 


\section{LINEAR TYPE:}

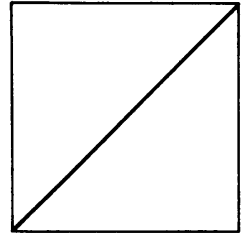

$p=0, m=1$

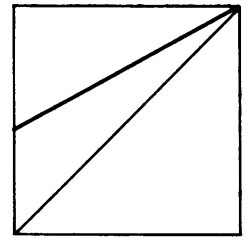

$0<p<1, m<1$

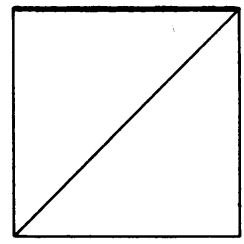

$p=1, m=0$

\section{CONVEX TYPE:}

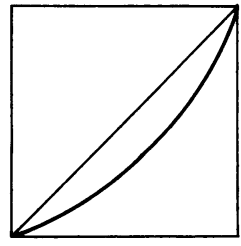

$p=0, m>1$

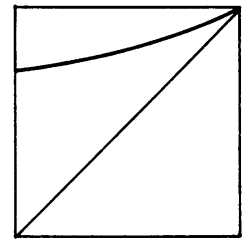

$0<p<1, m<1$

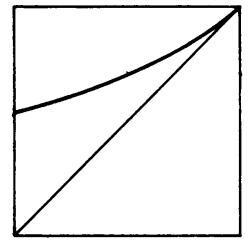

$0<p<1, m=1$

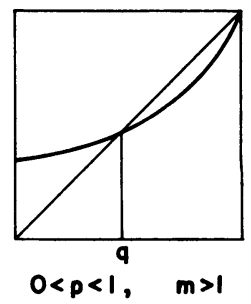

CONCAVE TYPE:

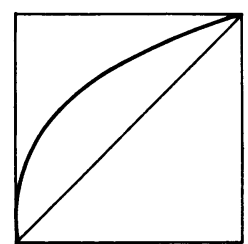

$p=0, m<1$

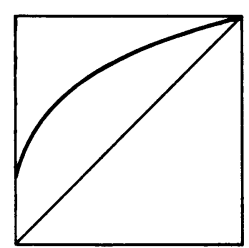

$0<p<1, m<1$

P-O-TYPE:
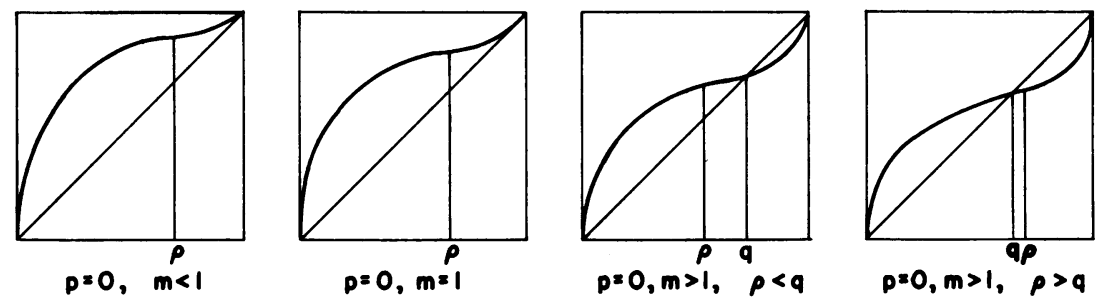

$p$-p-TYPE.
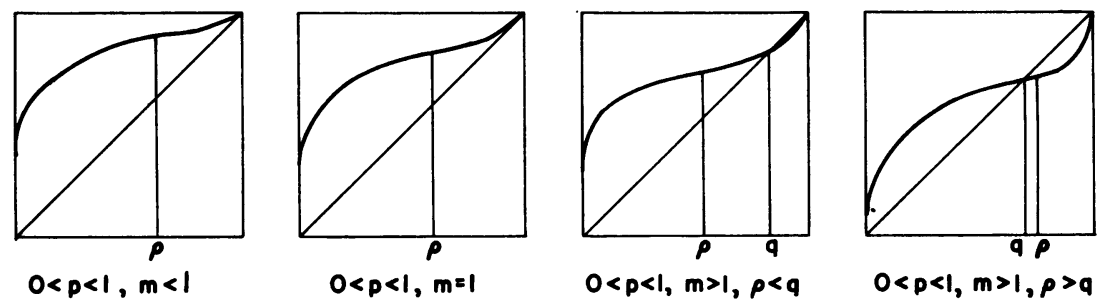

FIG. 1.-Graphs of typical probability generating functions. 
Proof: Let $\alpha, \beta, \lambda$ be nonnegative constants; let $Y$ be Poisson-distributed with parameter $\lambda$, and let $X=\alpha Y+\beta$. It can then be seen, inspecting the probability generating function $f$ of $X$ and the first two derivatives of $f$, that the parameters $\alpha, \beta, \lambda$ can be adjusted in such a way that $f$ is of any desired type except linear. The linear types correspond to $P(X=0)=1-P(X=1)=\rho$.

3. Iterates and Compositions of Probability Generating Functions.-Iterates of pgf's play a basic role in the investigation of branching sequences (refs. 2 and 3). If the "branching conditions" change from generation to generation, then these iterates have to be replaced by compositions of different pgf's.

TheоReм 3.1. The types of probability generating functions as listed in Theorem 1.1 are invariant under iteration. More precisely, if $f$ and $g$ have one of the following properties in common, then their composition $f \circ g$ has this same property:

(a) linear type, convex type, concave type, $\mathrm{p}$-type;

(b) 0-type, p-type;

(c) $m<1, m=1, m>1$;

(d) existence of $q$ with $f(q)=q$.

Proof: All these assertions involve the values of $f \circ g$ and of its first two derivatives and can be shown using

$$
\begin{aligned}
& (f \circ g)^{\prime}(s)=f^{\prime}(g(s)) g^{\prime}(s) \\
& (f \circ g)^{\prime \prime}(s)=f^{\prime \prime}(g(s))\left(g^{\prime}(s)\right)^{2}+f^{\prime}(g(s)) g^{\prime \prime}(s) .
\end{aligned}
$$

For $(d)$ we give a direct proof, not involving the derivatives, which shows even a little more: If $s<\min \left(q_{f}, q_{\sigma}\right)$, then $g(s)>s, f(s)>s$ and thus $f(g(s)) \geq s$; conversely, $s>\max \left(q_{f}, q_{g}\right)$ will imply $f(g(s))<s$. Thus, $q_{\text {fog }}$ exists and is intermediate between $q_{f}$ and $q_{0}$.

The author does not know whether the subtypes $\rho<q, \rho=q, \rho>q$ of the $\rho$ type with $m>1$ are invariant under composition; however, under iteration even a stronger statement can be made.

THEOREM 3.2. If $f(q)=q, 0<q \leq 1$, then for all iterates $f_{n}$ we have $f_{n}(q)=$ $q$; moreover, $f_{n}(s) \rightarrow q$ for $0<s<1$. If $f(1)=1$ is the only solution of $f(s)=s$, then $\lim _{n \rightarrow \infty} f_{n}(s)=1$; if $f(0)=0$ and $f(1)=1$ are the only solutions of $f(s) \rightarrow s$, then $f_{n}(s) \rightarrow 0$ for all $s \in\left[0,1\left[\right.\right.$ in the convex case, or $f_{n}(s) \rightarrow 1$ for all $\left.\left.s \epsilon\right] 0,1\right]$ in the concave and $\rho$-type cases.

If $f$ is of the $\rho$-type with $m>1$, then if $f(q)=q$ and $f_{n}^{\prime \prime}\left(\rho_{n}\right)=0$, the following is true:

(i) if $\rho_{1}=q$, then $q=\rho_{1}=\rho_{2}=\ldots$;

(ii) if $\rho_{1}<q$, then $q>\rho_{1}>\rho_{2}>\ldots$;

(iii) if $\rho_{1}>q$, then $q<\rho_{1}<\rho_{2}<\ldots$.

Proof: The first part of the theorem is shown exactly as for probability generating functions of integer-valued variables (see, e.g., ref. 2, pp. 6 and 7). To prove the second part, we need two versions of (3.2).

$$
\begin{aligned}
& f_{n+1}^{\prime \prime}(s)=f_{n}^{\prime \prime}(f(s))\left(f^{\prime}(s)\right)^{2}+f_{n}^{\prime}(f(s)) f^{\prime \prime}(s), \\
& f_{n+1}^{\prime \prime}(s)=f^{\prime \prime}\left(f_{n}(s)\right)\left(f_{n}^{\prime}(s)\right)^{2}+f^{\prime}\left(f_{n}(s)\right) f_{n}^{\prime \prime}(s) .
\end{aligned}
$$

Statement $(i)$ follows readily from either one of these relations. Next, show (ii), putting $s=\rho$, in (3.3) it is seen that $\rho_{n}<\rho_{1} ; n=2,3, \ldots$; then assuming $\rho_{n}<$ 
$\rho_{n+1}$ for some $n$ and using (3.4) first with $s=\rho_{n+1}$ and then with $s=\rho_{n}$, it follows that $\rho_{n-1}<\rho_{n}$ which leads to a contradiction. The proof of (iii) is analogous.

Remark 3.1: The probabilistic meaning of $p$ and $m$ is obvious; $q$ is the probability $P\left(Z_{n} \rightarrow 0 \mid Z_{0}=1\right)$ for a branching sequence with basic probability generating function $f$ (refs. 1 and 2). It is not clear, however, what the probabilistic meaning of $\rho$ could be.

* This paper was prepared with partial support of the National Institutes of Health, grant 2 RO 1 GM 10525-04.

${ }^{1}$ Bühler, W. J., "Limit theorems for branching processes," Ph.D. thesis, University of California, Berkeley, 1966.

${ }^{2}$ Harris, T. E., The Theory of Branching Processes (Berlin-Göttingen-Heidelberg: SpringerVerlag, 1963).

3 Jiřina, M., "Stochastic branching processes with continuous state space," Czech. Math. J., 8, 292-313 (1958). 